June 29, 2012

\title{
Laser Mode Behavior of the Cassini CIRS Fourier Transform Spectrometer at Saturn
}

John C. Brasunas

NASA Goddard Space Flight Center, Greenbelt, MD 20771, USA

HIGHLIGHTS

- Cassini CIRS Fourier transfrom spectrometer in orbit about Saturn

- Wavelength calibration via temperature-stabilized laser diode

- Laser mode may change after instrument turn-off/turn-on cycle

- Characterize mode change via Mylar features due to beam splitter substrate

\begin{abstract}
The CIRS Fourier transform spectrometer aboard the NASA/ESA/ASI Cassini orbiter has been acquiring spectra of the Saturnian system since 2004. The CIRS reference interferometer employs a laser diode to trigger the interferogram sampling. Although the control of laser diode drive current and operating temperature are stringent enough to restrict laser wavelength variation to a small fraction of CIRS finest resoluton element, the CIRS instrument does need to be restarted every year or two, at which time it may start in a new laser mode. By monitoring the Mylar absoption features in uncalibrated spectra due to the beam splitter Mylar substrate, it can be shown that these jumps are to adjacent modes and that most of the eight-year opeation so far is restricted to three adjacent modes. For a given mode, the wavelengh stability appears consistent with the stability of the laser diode drive current and operating temperature.
\end{abstract}

Keywords:

Fourier transform spectrometer

Wavelength calibration

Laser mode

Mylar

john.c.brasunas@nasa.gov

$+1301.286 .3488$

+1301.286 .1683 (fax) 


\section{Introduction}

The NASA-ESA-ASI Cassini-Huygens space mission was launched in 1997, arriving at the Saturnian system in 2004. The Cassini orbiter continues to operate and return data to Earth. Among the remote-sensing instruments aboard Cassini is CIRS [1], a Fourier transform spectrometer (FTS) with a $50.8 \mathrm{~cm}$ input telescope and a dual FTS configuration with a common moving-mirror mechanism [Fig. 1]. The far-infrared (FIR) FTS covers the $10-600 \mathrm{~cm}^{-1}$ spectral region with the FP1 (focal plane 1) thermopile detectors at $170 \mathrm{~K}$, isothermal with most of the optics. The mid-infrared (MIR) FTS covers the 600-1100 and 1100-1400 $\mathrm{cm}^{-1}$ regions with two HgCdTe focal planes (FP3 and FP4). FP3 and FP4 are cooled to near $80 \mathrm{~K}$ with a passive radiator. Most electronics are housed separately at $300 \mathrm{~K}$ on the Cassini spacecraft. The spectral resolution is selectable by command, ranging from 0.5 to $15.5 \mathrm{~cm}^{-1}$, corresponding to scan times of 50 and $2 \mathrm{~s}$, respectively (round-trip).

\section{Cassini CIRS FTS}

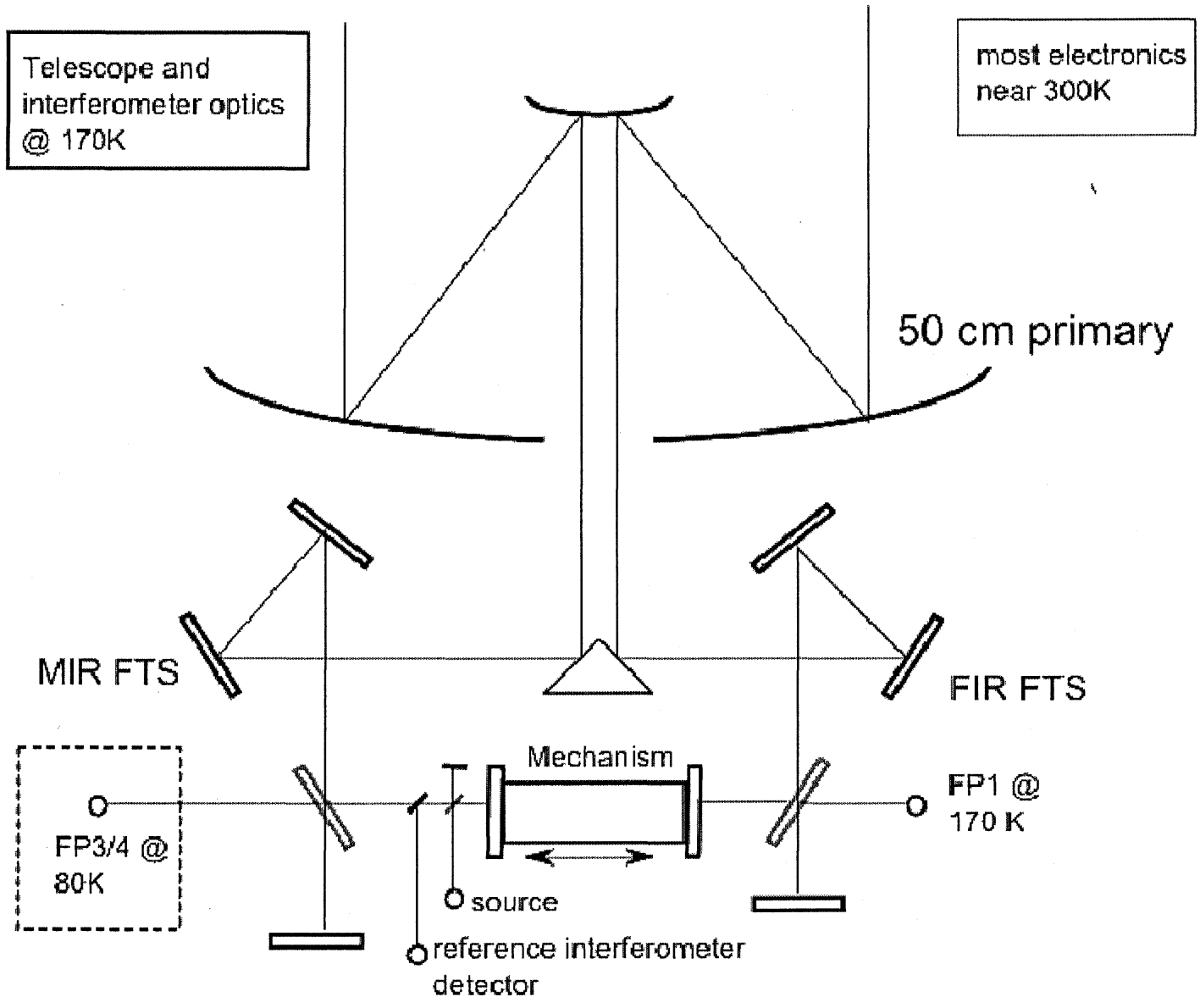

Fig. 1 cIRS optical-mechanical layout (not to scale) 


\section{Steps taken to stabilize CIRS wavelength scale}

To make data calibration practical, it is necessary to make the sampling of the FTS raw data, the interferogram, as repeatable as possible from scan to scan. As is common with the FTS, CIRS includes a so-called reference interferometer that utilizes the MIR moving mirror. The reference interferometer includes a light-emitting diode, to signal the start of the forward scan, and a laser to trigger the sampling of FP1, 3 and 4. As the CIRS laser is a single-mode AlGaAs laser diode SDL 5601 [2], its wavelength (nominally $783 \mathrm{~nm}$ at $170 \mathrm{~K}$ ) and thus the CIRS sampling comb stability is limited by the stability of the temperature of the laser diode [Fig. 2] and by the stability of the laser drive current [Fig. 3]

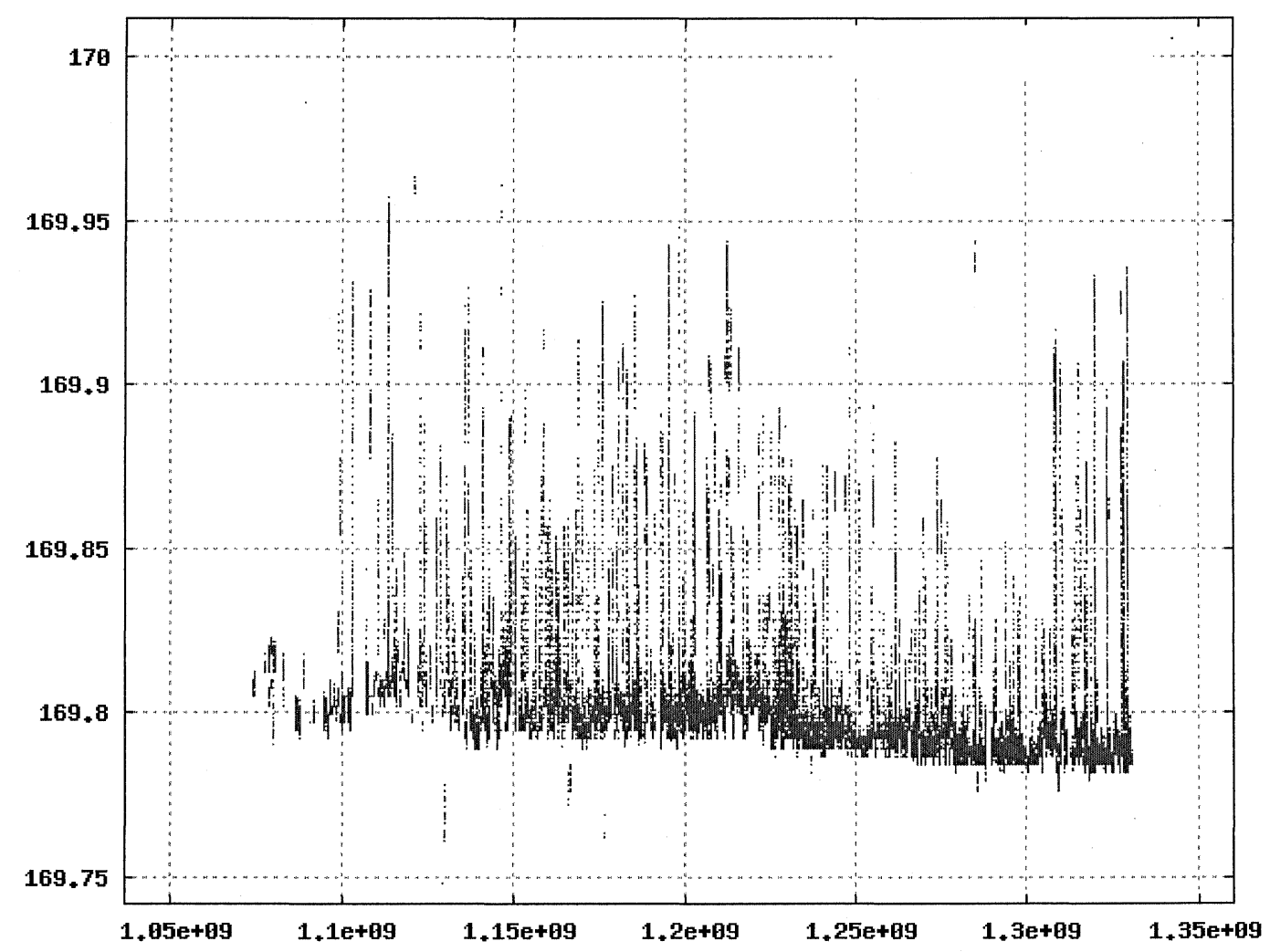

Fig. 2 Laser temperature (K) vs. UTC time (s) from 2004 to the present ( 50 million $s \sim 18$ months)

The long-term temperature drift over $\sim 8$ years appears to be about 0.01 to $0.02 \mathrm{~K}$. With a typical between-mode-hops laser coefficient of $\sim 0.08 \mathrm{~nm} / \mathrm{K}$, the long-term laser wavelengh drift should not exceed $\sim 0.0016 \mathrm{~nm}$, with a shorter term variability of $0.1 \mathrm{~K}$ corresponding to $0.008 \mathrm{~nm}$. Given a characteristic laser driver current effect (a change of $20 \mathrm{~mA}$ corresponding to $\sim 3 \mathrm{~K}$ temperature change), the $0.016 \mathrm{~mA}$ quantization level of the laser current corresponds to $\sim 0.0002 \mathrm{~nm}$. These shifts and the typical laser mode spacing are compared with the CIRS spectral resolution in Table 1. 


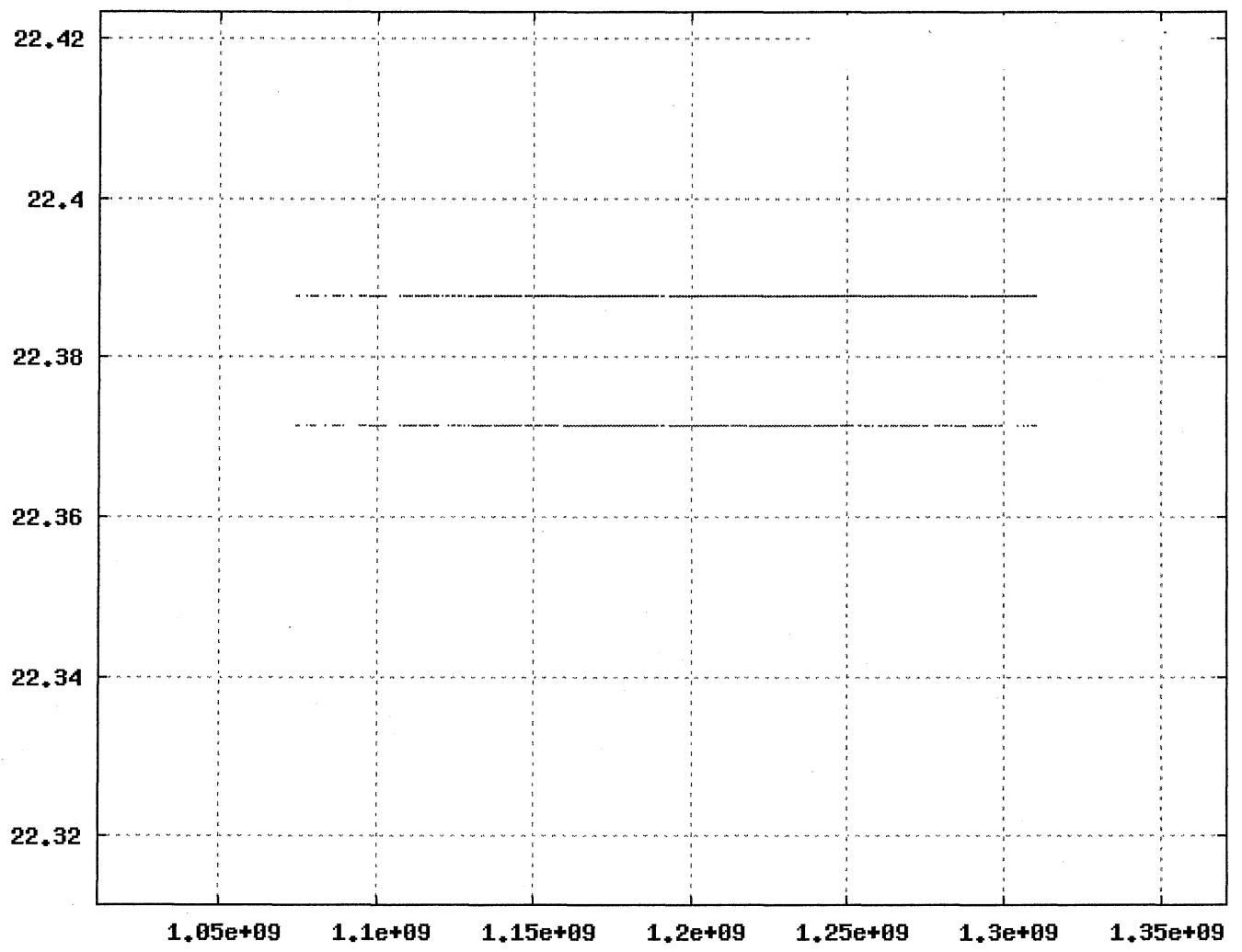

Fig. 3 Laser drive current (mV) Vs. UTC (s) over course of the mission

Table 1 Laser diode characteristics compared with CIRS spectral resolution

\begin{tabular}{|l|l|l|l|l|}
\hline $\begin{array}{l}\text { Environmental } \\
\text { Factor }\end{array}$ & Variation & Limiting factor & $\begin{array}{l}\text { Laser line } \\
\text { (nm) }\end{array}$ & $\begin{array}{l}\text { Fractional } \\
\text { change }\end{array}$ \\
\hline Thermal -2 minutes & $0.01 \mathrm{~K}$ & thermal inertia & 0.0008 & $1 / 10^{6}$ \\
\hline Thermal - 24 hours & $0.1 \mathrm{~K}$ & active control & 0.008 & $1 / 10^{5}$ \\
\hline $\begin{array}{l}\text { Thermal - year-to-year } \\
\text { trend over } 8 \text { years }\end{array}$ & $\sim 0.02 \mathrm{~K}$ & aging? & 0.0016 & $2 / 10^{6}$ \\
\hline Bias current stability & $0.016 \mathrm{~mA}$ & quantization & 0.0002 & $1 /\left(4 \times 10^{6}\right)$ \\
\hline Mode spacing $(\Delta \mathrm{m}=1)$ & integer spacing & cavity length & $\sim 0.2$ & $1 / 4000$ \\
\hline FP1 spectral resolution & $0.5 \mathrm{~cm}^{-1}$ & scan length & ---- & $1 / 1200$ \\
\hline FP4 spectral resolution & $0.5 \mathrm{~cm}^{-1}$ & scan length & ---- & $1 / 2800$ \\
\hline
\end{tabular}




\section{Evidence of changing laser mode over the course of the Cassini mission}

Table 1 demonstrates that the CIRS environmental stability is good enough to keep the laser wavelength change to a small fraction of the highest relative spectral resolution at the high ends of the various CIRS focal planes. It is also important to maintain laser diode operation within a single mode. For the CIRS flight model lasers at the $170 \mathrm{~K}$ operating temperature, the lasers remain in a single mode operation over a wide range of drive currents. While the moving mirror is scanning forward, the reference laser fringes trigger the $\mathrm{A} / \mathrm{D}$ sampling circuit at $\sim 1 \mathrm{KHz}$. A rectified and low-pass filtered version of the laser signal (sampled every second) is stored in the housekeeping data, keeping a record of the envelope of the interferogram signal from the reference interferometer. The envelope signal at 3 seconds after start of forward scan is shown in Fig. 4.

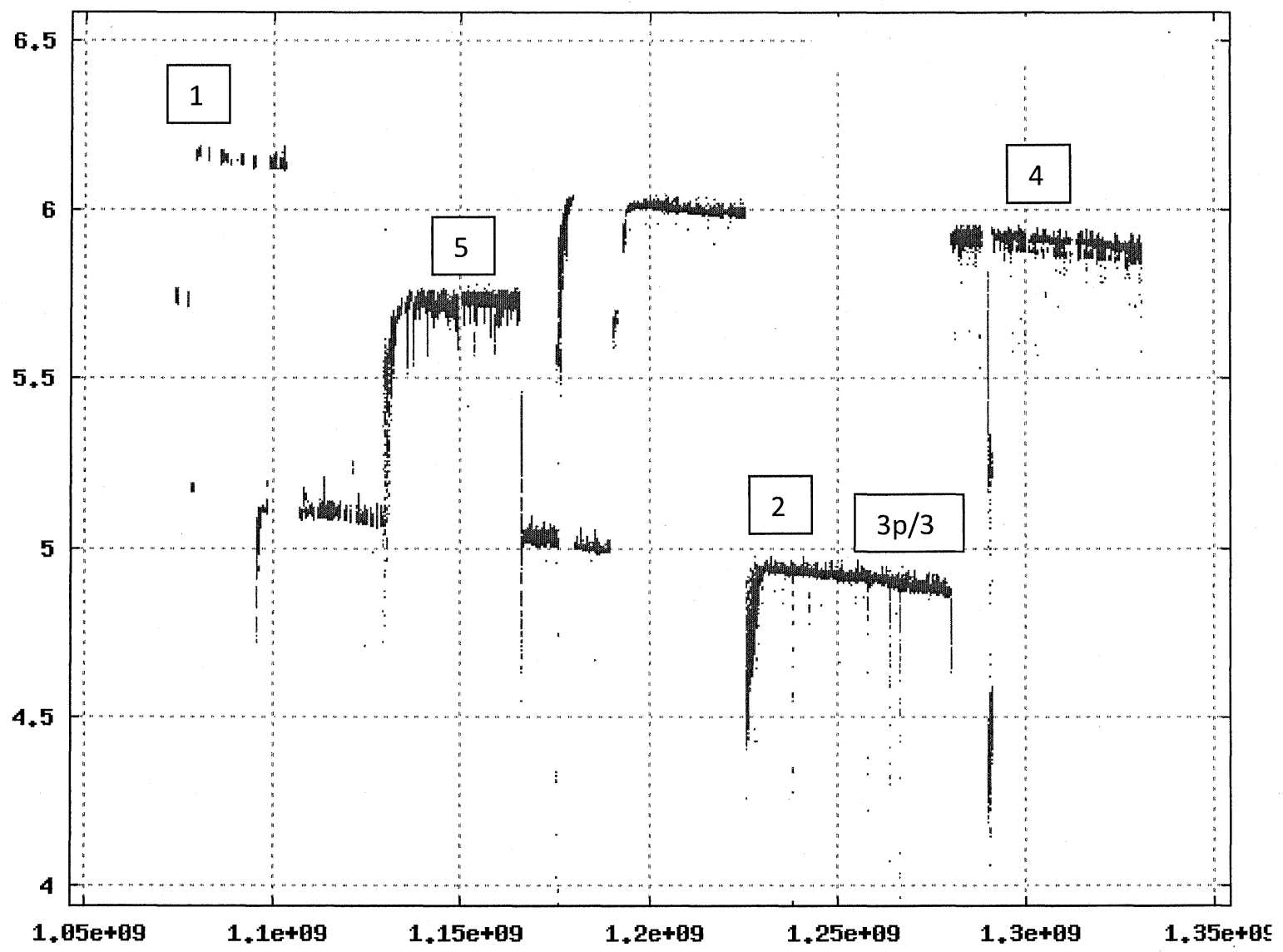

Fig. 4 Reference laser envelope signal (V) at 3s after start of forward scan, vs. UTC (s) over course of mission.

The jumps in Fig. 4 are apparently mode hops. These mode hops correspond to times when the CIRS instrument needed to be turned off or switched to the sleep mode. When the laser is turned back on, single-mode operating is acquired shortly, but without any guarantee that the mode after turn-on will be the same as the mode before turn-off. From the above figure, it appears intervals 1 and 4 are in one mode, intervals 2,3 , and $3 p$ (rime) are in another. It is not known if the slope within modes corresponds to an 
actual drift in wavelength, nor it is understood why interval 5 has a different slope at an atypical spacing relative to the other modes. Nor is it clear what the relationship is between laser envelope signal and laser wavelength, other than to indicate when the laser mode has changed.

\section{Method to quantify laser modes: spacing, and within-mode wavelength stability}

CIRS has an on-board calibration target, but it is a broad-band thermal target for intensity calibration. There is no on-board wavelength calibration target, and the reference laser itself is not locked to any atomic or molecular feature. It is possible to calibrate the wavelength by comparing observed and theoretical spectra of atmospheric features on Saturn or Titan. However this is time-consuming and requires careful attention to detail in a multi-layer atmospheric model with a priori unknown distributions of temperature and chemical abundances. Are there any useful spectral signals in the calibration targets themselves? The answer appears to be yes for FP1, but not for FP3 or FP4. The FIR FTS (Fig. 1) is a polarizing-type FTS; the beam-splitter is actually a wire-grid on a Mylar substrate. Fig. 5 shows the average of $\sim 5,000$ un-calibrated FP1 spectra taken at the highest spectral resolution, with Mylar features marked as $\mathrm{A}, \mathrm{B}$ and $\mathrm{C}[3]$.

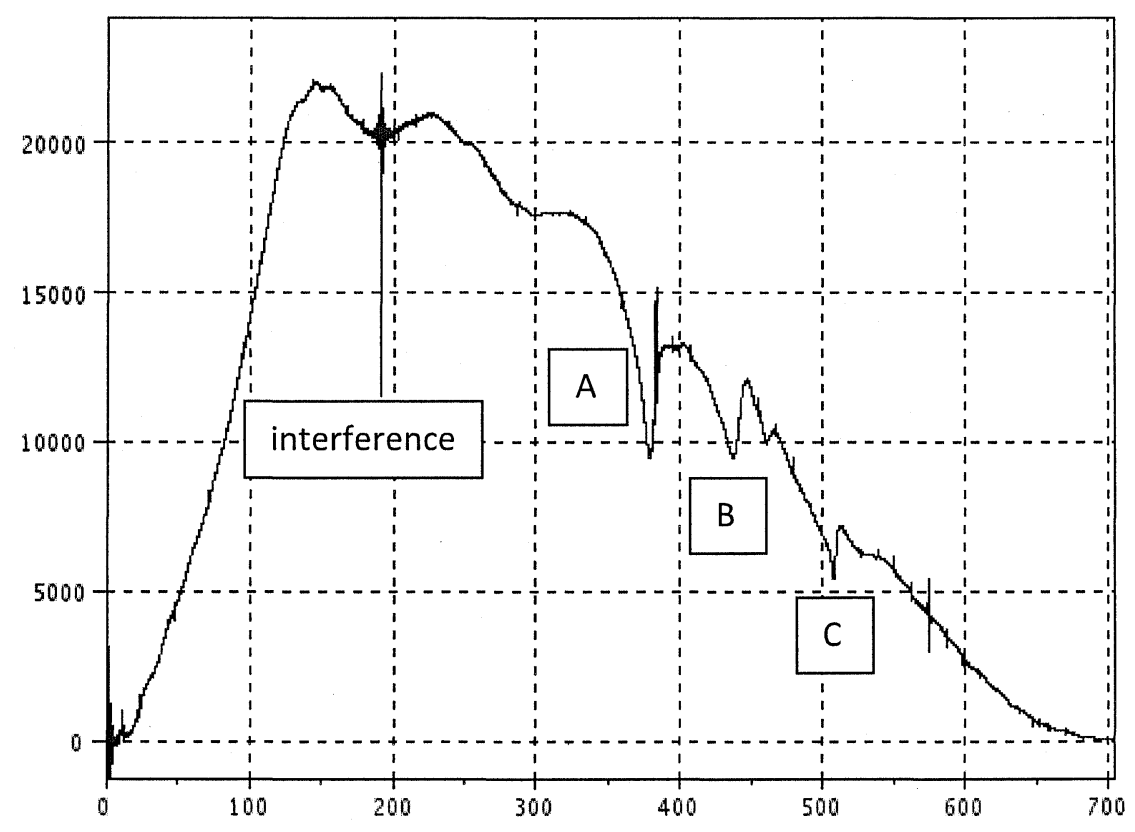

Fig. 5 FP1, showing Mylar and interference features; un-calibrated spectrum vs. $\mathrm{cm}^{-1}$

The idea is to use the Mylar features to study the laser mode hops; it is not expected that the Mylar features would exhibit sudden changes in position. There is also an interference feature near 200 $\mathrm{cm}^{-1}$, corresponding to $8 \mathrm{~Hz}$. This features in due to digital communications between CIRS and the Cassini spacecraft, which add coherently scan-to-scan because the repeat cycle of the CIRS scans is 
locked to a multiple of the spacecraft clock (especially near the beginning of the mission). The interference fundamental has harmonics near $380 \mathrm{~cm}^{-1}$ and $570 \mathrm{~cm}^{-1}$, making the use of Mylar feature A problematic. Fig. 6 shows the Mylar features in greater detail.

The central idea then of this paper is to create averages of $\sim 4,000$ to 5,000 un-calibrated FP1 spectra taken at the highest spectral resolution, for each of the numbered, $\sim$ month-long-periods shown in Fig. 4. The un-calibrated spectra are interpolated to a resolution of $\Delta \sigma \sim 0.0326 \mathrm{~cm}^{-1}$. Taking any two periods, with corresponding spectral averages $\operatorname{spect} 1(\sigma)$ and $\operatorname{spect} 2(\sigma)$, compute the root-mean square difference diffn:

$$
\operatorname{diffn}=\Sigma((\operatorname{spect} 1(\sigma)-\operatorname{spect} 2(\sigma+m \Delta \sigma)) * * 2 /(\operatorname{spect} 1(\sigma) * \operatorname{spect} 2(\sigma+m \Delta \sigma)))
$$

where the spectra from the two periods are offset an integral number $\mathrm{m}(+/-32)$ of resolution elements $\Delta \sigma$, and the limits of summation correspond to the two windows for Mylar features B and C.

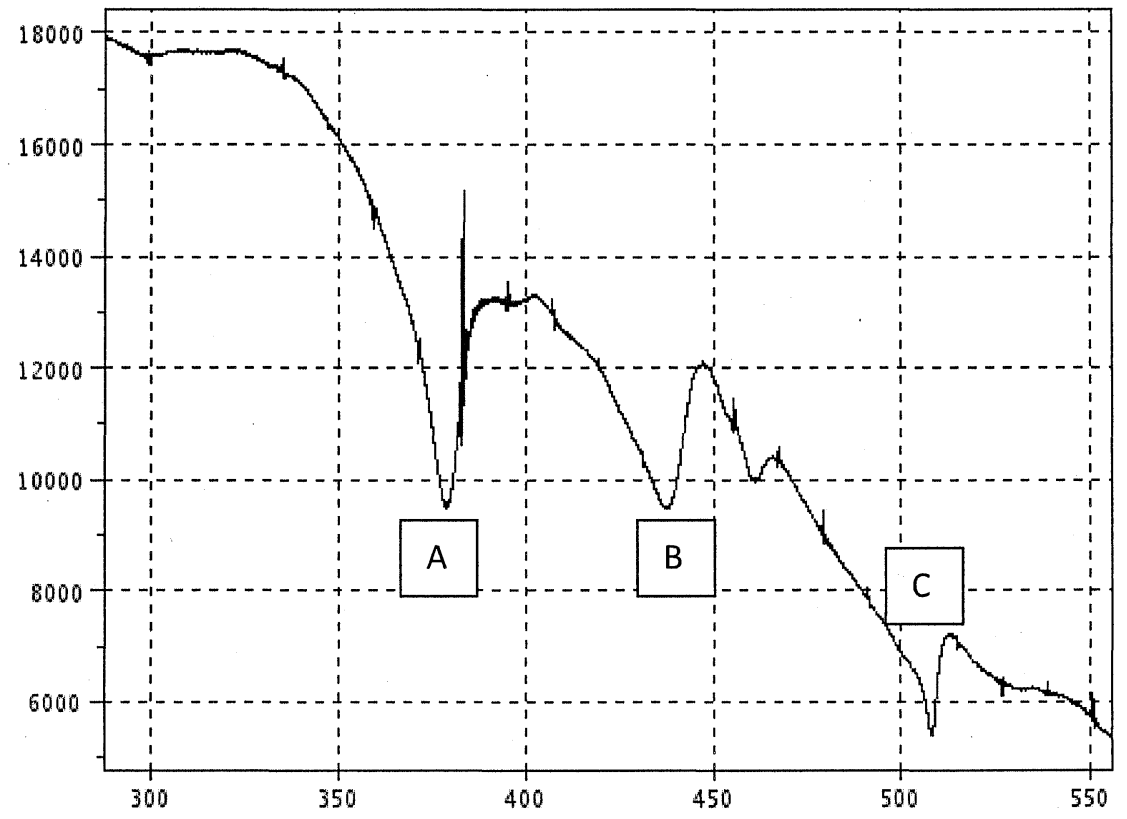

Fig. 6 Zoom in on Mylar features in FP1, un-calibrated spectrum vs. $\mathrm{cm}^{-1}$.

Mylar feature B: $\quad 420$ to $450 \mathrm{~cm}^{-1}$

Mylar feature C: $\quad 500$ to $515 \mathrm{~cm}^{-1}$.

A typical plot of diffn for window $\mathrm{C}$ is shown in Fig. 7 (the offsets $0,+/-32$ have been re-mapped to $1: 65$ ). Feature $C$ has a stronger sensitivity to changes in wavelength, with a 0.95 change in diffn for this range of offsets, versus $\sim 0.35$ change for feature $\mathrm{B}$. An offset of one unit of shift corresponds to a 
wavelength change of $\sim 1 / 13,300$ for $\mathrm{B}$ and $1 / 15,600$ for $\mathrm{C}$. A parabola is fit to the minimum to define the location of the minimum with sub-integral resolution.

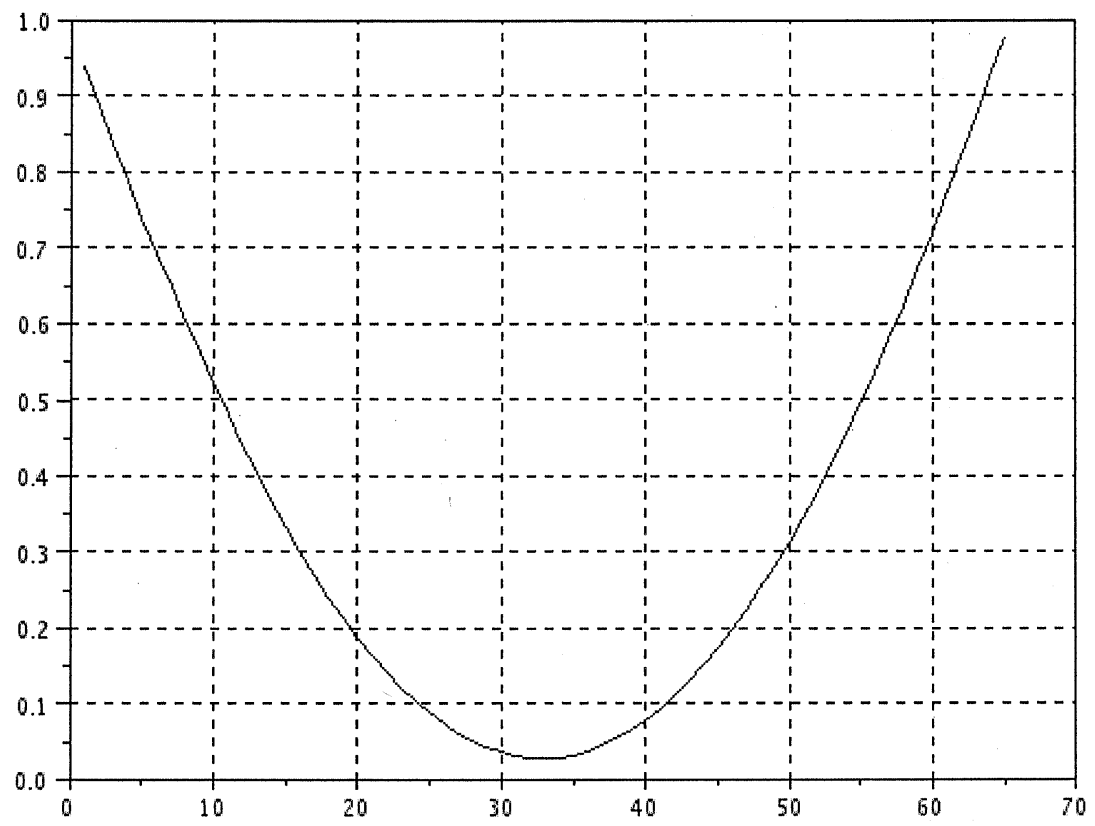

Fig. 7 diffn for Mylar feature C vs. shift ('33' corresponds to no shift) 


\section{Results and conclusion}

The FP1 data details for the six periods of Fig. 4 are given in Table 2. SCET (SpaceCraft Event Time) is another name give to spacecraft UTC time. RTI (real-time interrupt) is the trigger-signal supplied by the Cassini spacecraft at $8 \mathrm{~Hz}$. The longest CIRS scans are $\sim 50 \mathrm{~s}$ or $\sim 400 \mathrm{RTI}$. ZPD (zero path difference) is the location of the FP1 zpd relative to the reference interferometer start of scan signal. AMP is the interferogram amplitude at ZPD. ZPD and AMP are used to select either deep-space scans or scans with weak external targets, so as not to mask the Mylar feature. NPTS is the minimum number of interferogram samples available for long scans for each period. All theses FP1 scans are for no coadding, and additional selection on a criterion $\mathrm{NOISE}=1$ has been made to reject some known problems with the data.

Table 2 FP1 data details for various periods to be analyzed for changes in laser wavelength

\begin{tabular}{|l|l|l|l|l|l|l|}
\hline Period & 1 & 2 & 3 & $3 p$ & 4 & 5 \\
\hline Month(s) & Late 2004 & $\begin{array}{l}\text { January } \\
2009\end{array}$ & June 2010 & May 2010 & $\begin{array}{l}\text { January } \\
2011\end{array}$ & $\begin{array}{l}\text { June-July } \\
2006\end{array}$ \\
\hline SCET & 1080777600 & 1230768000 & 1275350400 & 1272672000 & 1293840000 & 1149120000 \\
& 1101859200 & 1233446400 & 1277942400 & 1275350400 & 1296518400 & 1154390400 \\
\hline RTI & 400 & 401 & 401 & 401 & 401 & 401 \\
\hline ZPD & $89.4-89.48$ & $89.37-89.45$ & $89.38-89.46$ & $89.38-89.46$ & $89.39-89.47$ & $89.36-89.42$ \\
\hline AMP & -11000 & -11000 & -11000 & -11000 & -11000 & -11000 \\
& -10500 & -10500 & -10500 & -10500 & -10500 & -10500 \\
\hline NPTS & 2755 & 2720 & 2718 & 2720 & 2719. & 2720 \\
\hline Temperature & 169.795 & 169.79 & 169.78 & 169.78 & 169.78 & 169.79 \\
(K) & 169.82 & 169.88 & 169.86 & 169.88 & 169.86 & 169.89 \\
\hline Scans & 4473 & 3949 & 2828 & 3575 & 5065 & 3692 \\
\hline
\end{tabular}

The estimates of laser shifts are given in Table 3, comparing pairs of periods to estimate either the uncertainty (adjacent periods), mode shifts, or wavelength trends within a mode. The interferograms are separately processed for each pair-wise comparison, trimming the interferograms in both periods to a common, maximum possible length. Un-calibrated spectra are produced, averaged over each period, and the minimum of diffn is estimated via parabolic interpolation on diffn. Recall that a minimum located at 33 corresponds to no shift, and the unit of shift (a 34 instead of a 33) corresponds to a shift of 0.0326 $\mathrm{cm}^{-1}$. The uncertainty in the shift estimate appears to be $\sim 0.3$ for the 500-515 window, and somewhat more for the 420-450 window. The mode shift seems to be $\sim 2.8$ (period 4 to 3 ), and 3.0 (period 3 to 5 ). Thus the modes appear to be equally spaced as they should be, and the implied wavelength change is $1 / 5000$ to $1 / 4400$, quite close to the nominal laser mode spacing as reported in Table 1 . Thus these appear to be jumps between adjacent modes $(\Delta \mathrm{m}=1)$. As for the within mode trends, Fig. 4 shows a 1 to 4 change $\sim 20 \%$ of the mode jump at 4 to 3 . This would be a shift of 0.6 if Fig. 4 is assumed proportional to the laser wavelength. This is not seen in the 1 to 4 comparison for the $500-515 \mathrm{~cm}^{-1}$ comparison, and is within the noise for the 420-450 window. Not seeing a within-mode laser trend at this level is consistent with the stability of instrument temperature and laser drive current, which would indicate a wavelength trend less than $1 \%$ of the mode spacing over the course of eight years. 
Table 3 Estimates of laser wavelength changes between various pairs of time periods

\begin{tabular}{|l|l|l|l|l|l|}
\hline Comparison & 3 vs. 3p & 4 vs. 3 & 3 vs. 5 & 2 vs. 3 & 1 vs. 4 \\
\hline Purpose & uncertainty & mode change & mode change & trend in mode & trend in mode \\
\hline $\begin{array}{l}\mathbf{4 2 0 - 4 5 0} \mathbf{~ c m}^{-1} \\
\text { window }\end{array}$ & & & & & \\
\hline Minimum location & 32.48 & 35.8 & 35.45 & 32.45 & 32.4 \\
\hline Minimum of diffn & 0.0032 & 0.0035 & 0.032 & 0.014 & 0.028 \\
\hline $\begin{array}{l}\mathbf{5 0 0 - 5 1 5} \text { cm }^{-1} \\
\text { window }\end{array}$ & & & & & \\
\hline Minimum location & 33.3 & 35.8 & 36.6 & 33.13 & 32.9 \\
\hline Minimum of diffn & 0.0055 & 0.006 & 0.03 & 0.008 & 0.028 \\
\hline $\begin{array}{l}\text { Conclusion } \\
\text { Uncertainty } \sim \\
\Delta \mathrm{m}=0.1\end{array}$ & $\Delta \mathrm{m}=1$ & $\Delta \mathrm{m}=1$ & $\begin{array}{l}\text { less than Fig. } 4 \\
\text { implies }\end{array}$ & $\begin{array}{l}\text { less than Fig. } 4 \\
\text { implies }\end{array}$ \\
\hline
\end{tabular}

In conclusion, the CIRS reference interferometer provides an indication of a change in laser mode after CIRS instrument restart. However the relation between laser wavelength and this indicator is not monotonic. By using Mylar absorption features originating in the beam splitter substrate, it is possible to identify spectral features present in all the intensity calibration scans of one of the infrared focal planes. From observed shifts of these Mylar features it can be shown these mode changes are jumps among three, equally spaced levels. It is also possible to confirm within-mode wavelength stability at a level consistent with the stability of the laser diode drive current and operating temperature over an eightyear period.

\section{Acknowledgments}

The author acknowledges support from NASA's Cassini mission and from the Cassini CIRS calibration team.

\section{References}

[1] F.M. Flasar, V.G. Kunde, M.M. Abbas, R.K. Achterberg, P. Ade, A. Barucci, B. Bezard, G.L. Bjoraker, J.C. Brasunas, S. Calcutt, R. Carlson, C.J. Cesarsky, B.J. Conrath, A. Coradini, R. Courtin, A. Coustenis, S. Edberg, S. Edgington, C. Ferrari, T. Fouchet, D. Gautier, P.J. Gierasch, K Grossman, P. Irwin, D.E. Jennings, E. Lellouch, A.A. Mamoutkine, A. Marten, J.P. Meyer, C.A. Nixon, G.S. Orton, T.C. Owen, J.C. Pearl, R. Prange, F. Raulin, P.L Read, P.N. Romani, R.E. Samuelson, M.E. Segura, M.R. Showalter, A.A. Simon-Miller, M.D. Smith, J.R. Spencer, L.J. Spilker, F.W. Taylor, Exploring the Saturn system in the thermal infrared: the composite infrared spectrometer, Space Science Reviews, 115 (2004), 169-297.

[2] A.J. Martino, D.M. Cornwell, Reference interferometer using a semiconductor laser/LED reference source in a cryogenic Fourier-transform spectrometer, Proc. SPIE 3435 (1998) 343561.

[3] D.G. Johnson, K.W. Jucks, W.A. Traub, K.V. Chance, Smithsonian stratospheric far-infrared spectrometer and data reduction system, J. Geoophys. Res. 100 (1995), 3091-3106. 


\section{Captions}

Fig. 1 CIRS optical-mechanical layout (not to scale)

Fig. 2 Laser temperature (K) vs. UTC time (s) from 2004 to the present (50 million s 18 months)

Fig.3 Laser drive current (mV) Vs. UTC (s) over course of the mission

Fig.4 Reference laser envelope signal (V) at 3s after start of forward scan, vs. UTC (s) over course of mission.

Fig. 5 FP1, showing Mylar and interference features; un-calibrated spectrum vs. $\mathrm{cm}^{-1}$

Fig.6 Zoom in on Mylar features in FP1, uncalibrated spectrum vs. $\mathrm{cm}^{-1}$.

Fig.7 diffn for Mylar feature C vs. shift ('33' corresponds to no shift) 